\title{
Obtenção de carvão ativado a partir do resíduo fuligem de candeia
}

\author{
Obtention of activated carbon from \\ candeia soot residue
}

\author{
Pedro Barroso Gomes ${ }^{1}$, Maria Lúcia Bianchi ${ }^{1}$, \\ Fabiano Magalhães ${ }^{1}$
}

\author{
${ }^{1}$ Universidade Federal de Lavras, Departamento de Química, Praça Prof. Edmir Sá Santos s/n, CEP: $37200-$ \\ 900, Lavras, MG, Brasil \\ e-mail:pedrob.gomes@hotmail.com.br
}

\section{RESUMO}

Neste trabalho, o resíduo fuligem de candeia foi utilizada para obter carvão ativado. A ativação foi investigada utilizando três reagentes $\left(\mathrm{NaOH}, \mathrm{H}_{3} \mathrm{PO}_{4}\right.$ e $\left.\mathrm{CO}_{2}\right)$. Os resultados de análise térmica e microscopia eletrônica de varredura mostram que os carvões ativados possuem maior estabilidade térmica e partículas mais regulares e menores quando comparados ao precursor. Medidas de área superficial mostraram que os carvões ativados com $\mathrm{NaOH}$ e $\mathrm{CO}_{2}$ apresentaram resultados iguais a 804 e $435 \mathrm{~m}^{2} \mathrm{~g}^{-1}$, respectivamente, e maior capacidade para adsorver o azul de metileno (222 e $118 \mathrm{mgg}^{-1}$, respectivamente). Os valores de capacidade de adsorção são cerca de 10,6 e 5,6 vezes maiores para os carvões ativados em comparação à capacidade de adsorção da fuligem. Os resultados demonstram o grande potencial de se obter carvão ativado a partir de fuligem residual.

Palavras-chave:Carvão ativado, fuligem, adsorção.

\begin{abstract}
In this study, candeia soot waste was used for the production of activated carbons. Activation was investigated using three reagents $\left(\mathrm{NaOH}, \mathrm{H}_{3} \mathrm{PO}_{4}\right.$ and $\left.\mathrm{CO}_{2}\right)$. The results of thermal analysis and scanning electron microscopy show that the activated carbons present higher thermal stability and more regular and smaller particles when compared with the precursor. Surface area measurements showed that $\mathrm{NaOH}$ and $\mathrm{CO}_{2}$ activated carbons present higher specific surface areas (804 and $435 \mathrm{~m}^{2} \mathrm{~g}^{-1}$, respectively) and higher abilities to adsorb methylene blue (222 and $118 \mathrm{mgg}^{-1}$, respectively). The adsorption capacity values of the activated carbons are nearly10.6 and 5.6 times higher than the value found for the candeia soot residue. The results demonstrate the great potential of obtaining activated carbons from residual soot.
\end{abstract}

Keywords: Activated carbon, soot, adsorption.

\section{INTRODUÇÃO}

Anualmente, milhões de toneladas de resíduos agroindustriais são gerados por processos industriais. Grande parte dos resíduos são biomassas de origem natural, apresentando potencial para diversas aplicações [1]. Porém, devido às limitações técnicas e econômicas, os resíduos gerados geralmente não são utilizada como matéria prima para obtenção de novos materiais e acabam sendo destinados a aterros ou utilizados como fonte de energia. A utilização dos resíduos para geração de energia é exercida por praticamente todas as indústrias de beneficiamento de produtos da agroindústria, já que esse setor necessita de uma grande demanda energética [2]. Durante a combustão da biomassa é gerada a fuligem, que é um resíduo sólido, rico em carbono, proveniente da pirólise incompleta [3]. Este resíduo é coletado em lavadores de gases e normalmente destinado a aterro industrial ou é aplicado no solo [4].

Segundo a Política Nacional de Resíduos Sólidos [5] a fuligem residual apresenta potencial de contaminação ambiental, afetando a qualidade do ar, do solo e da água. A presença de partículas finas de fuligem na atmosfera pode causar doenças respiratórias e circulatórias, tais como asma, hipertensão e câncer de pulmão. $\mathrm{Na}$ literatura encontram-se trabalhos que utilizam biomassa para obtenção de carvão ativado (CA) ou biocarvões, $[6,7]$ porém na etapa de carbonização perde-se o potencial energético destes materiais. [8] No caso da 
fuligem, a energia armazenada na biomassa é quase toda utilizada. Desta forma, investigar processos para obter carvão ativado a partir de fuligem se torna muito interessante e vantajoso. Sendo assim, neste trabalho a fuligem de candeia foi ativada utilizando diferentes reagentes e o potencial de adsorção dos carvões ativados obtidos foi investigado para adsorção do corante azul de metileno, utilizado como molécula modelo.

\section{MATERIAIS E MÉTODOS}

\subsection{Materiais}

A fuligem de candeia (FC) utilizada no estudo foi cedida por uma empresa de extração de alfa-bisabolol, situada no estado de Minas Gerais. Os reagentes $\mathrm{NaOH}, \mathrm{H}_{3} \mathrm{PO}_{4}$ e azul de metileno utilizados foram da marca Synth.

\subsection{Obtenção dos Carvões Ativados}

Nas ativações de caráter químico, o precursor foi impregnado com agente ativante $\left(\mathrm{NaOH}\right.$ ou $\left.\mathrm{H}_{3} \mathrm{PO}_{4}\right)$ e as misturas resultantes foram mantidas em agitação por $24 \mathrm{~h}$. Após evaporar o solvente os materiais foram aquecidos em forno tubular $\left(10{ }^{\circ} \mathrm{Cmin}^{-1}, 100 \mathrm{mLmin}^{-1}\right.$ de $\left.\mathrm{N}_{2}\right)$ até a temperatura de ativação. A Tabela 1 apresenta as condições experimentais utilizadas, selecionadas por meio de revisão na literatura [9, 10]. Após a etapa de ativação, o carvão ativado com $\mathrm{NaOH}$ foi lavado com solução de $\mathrm{H}_{3} \mathrm{PO}_{4}$. Em seguida utilizou-se água destilada para reduzir a acidez dos carvões até atingir pH neutro.

Tabela 1: Condições utilizadas nos processos de ativação química da fuligem.

\begin{tabular}{c|c|c|c}
\hline AGENTE ATIVANTE & PROPORÇÃO FC:ATIVANTE (m:m) & TEMPERATURA DE ATIVAÇÃO ('⿳) & TEMPO (h) \\
\hline $\mathrm{NaOH}$ & $2: 1$ & 750 & 1 \\
\hline $\mathrm{H}_{3} \mathrm{PO}_{4}$ & $5: 1$ & 550 & 2 \\
\hline
\end{tabular}

$\mathrm{Na}$ ativação física, a FC foi acondicionada em tubo de quartzo inserido em forno tubular e a mesma foi aquecida a $900{ }^{\circ} \mathrm{C}\left(10^{\circ} \mathrm{Cmin}^{-1}\right)$ por $1 \mathrm{~h}$ sob fluxo de $\mathrm{CO}_{2}\left(150 \mathrm{mLmin}^{-1}\right)$.

Os CA obtidos foram identificados como $\mathrm{CAOH}, \mathrm{CAH}$ e $\mathrm{CACO}$ para os materiais ativados com $\mathrm{NaOH}$, $\mathrm{H}_{3} \mathrm{PO}_{4}$ e $\mathrm{CO}_{2}$, respectivamente.

\subsection{Caracterização dos materiais}

Os CA e a FC foram caracterizados por análise termogravimétrica e análise térmica diferencial(TG e DTA Shimatzu-DTG-60AH, de 30 à $900{ }^{\circ} \mathrm{C}, 10{ }^{\circ} \mathrm{Cmin}^{-1} \mathrm{em}$ ar sintético.), análise elementar (CHN - Elementar Vario EL cube), microscopia eletrônica de varredura (MEV - LEO EVO 40 XVP, tensão de 20 KV) e área superficial especifica BET (QuantaChromeAsiQwin 2.0), com temperatura e tempo de desgaseificação iguais a $200{ }^{\circ} \mathrm{C}$ e $12 \mathrm{~h}$, respectivamente.

\subsection{Ensaios de adsorção}

As isotermas de adsorção foram registradas utilizando $10 \mathrm{mg}$ de amostra e $10 \mathrm{~mL}$ de solução de azul de metileno (AM) 10, 25, 50, 100, 250, 500 e $1000 \mathrm{mg} \mathrm{L}^{-1}$. As amostras permaneceram em contato por $24 \mathrm{~h}$ sob agitação. Em seguida a mistura foi centrifugada (5 min a $3000 \mathrm{rpm})$ e o sobrenadante coletado. A eficiência da adsorção foi monitorada pela descoloração do AM utilizando um espectrofotômetro na região do ultravioleta/visível (UV/vis -Micronal AJX-3000PC) no comprimento de onda de $665 \mathrm{~nm}$.

\section{RESULTADOS E DISCUSSÃO}

A Figura 1 apresenta os resultados das análises térmicas TG e DTA obtidas para os materiais estudados. Nota-se que a FC apresenta três perdas de massas referentes à desidratação $\left(30\right.$ a $\left.155{ }^{\circ} \mathrm{C}\right)$, decomposição de compostos orgânicos $\left(240-390^{\circ} \mathrm{C}\right)$ remanescentes na fuligem (carbonização incompleta durante sua formação) e oxidação do carbono $\left(390-530{ }^{\circ} \mathrm{C}\right)$ [8]. Estes eventos são acompanhados por um pico exotérmico largo (Fig. 1A). Os resultados de TG obtidos para os CA (Fig. 1B) mostram que houve um aumento na estabilidade térmica, principalmente para a amostra $\mathrm{CAH}$, onde a oxidação do carbono inicia em $440{ }^{\circ} \mathrm{C}$. É interessante notar que o perfil termogravimétrico do $\mathrm{CAOH}$ mostra que a decomposição do carbono ocorre em duas eta- 
pas $\left(320\right.$ a $505^{\circ} \mathrm{C}$ e 505 a $\left.660{ }^{\circ} \mathrm{C}\right)$ que são acompanhadas por dois eventos exotérmicos, observados na Fig.1C. Este comportamento pode estar relacionado com a presença de resíduos do agente ativante que não foram completamente removidos na etapa de lavagem. [11].
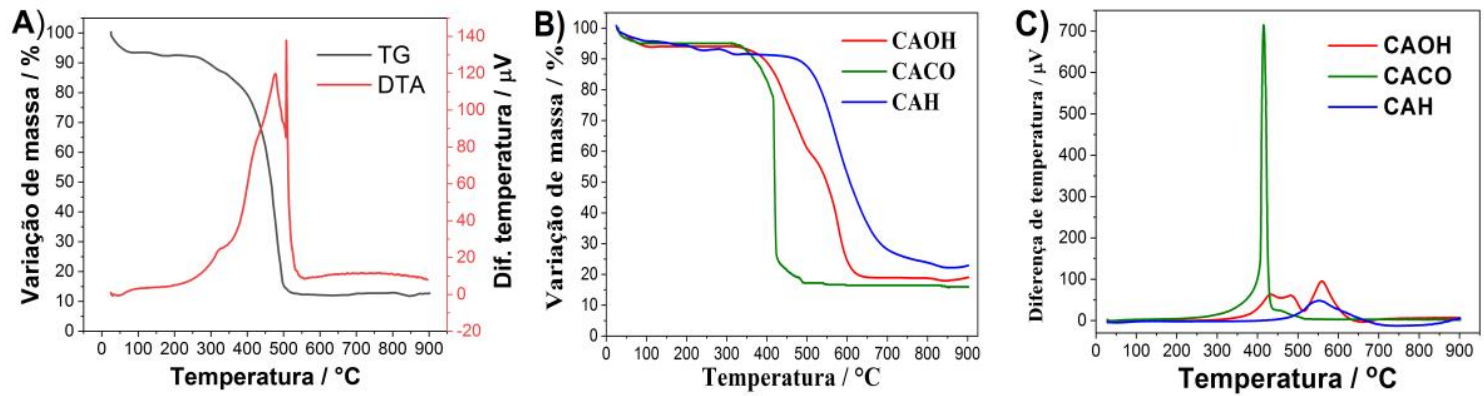

Figura 1: Curvas TG e DTA da FC (A) e dos CA (B e C) (ar sintético - $\left.100 \mathrm{mLmin}^{-1}, 10^{\circ} \mathrm{Cmin}^{-1}\right)$.

A Tabela 2 apresenta os resultados obtidos por análise elementar $\mathrm{CHN}$, e teores de carbono fixo e cinzas calculados pelos dados de TG.

Tabela 2: Resultados da análise elementar CHN e valores de carbono fixo e cinza obtido por TG.

\begin{tabular}{l|c|c|c|c|c|c}
\hline \multirow{2}{*}{ AMOSTRA } & \multicolumn{7}{c}{ TEOR/ \% } \\
\cline { 2 - 7 } & ${ }^{*} \mathbf{C}_{\text {fixo }}$ & $\mathbf{C}$ & $\mathbf{H}$ & $\mathbf{N}$ & $\mathbf{C} / \mathbf{H}$ & ${ }^{*}$ CINZAS \\
\hline FC & 80,3 & 82,4 & 2,2 & 1,0 & 37,4 & 13,1 \\
\hline CAOH & 75,2 & 78,7 & 1,0 & 0,9 & 78,7 & 20,0 \\
\hline CACO & 78,6 & 76,0 & 1,5 & 1,0 & 50,7 & 17,1 \\
\hline CAH & 72,2 & 70,1 & 1,7 & 1,0 & 41,2 & 24,3 \\
\hline
\end{tabular}

*Dados obtidos por TG

Os resultados mostram que os teores de carbono fixo determinados por TG e os teores elementares de carbono obtidos por análise elementar estão próximos, indicando que o precursor e os CA apresentam um alto teor de carbono fixo. A redução no teor de carbono observado para os CA em relação à FC está relacionada com o seu consumo durante o processo de ativação [12]. Nota-se também redução nos teores de $\mathrm{H}$ e $\mathrm{N}$ após ativação, o que certamente estão relacionados com a perda de grupos funcionais e compostos orgânicos voláteis durante o tratamento térmico. Nota-se que a relação $\mathrm{C} / \mathrm{H}$ aumentou na seguinte ordem: $\mathrm{CAH}<\mathrm{CA}$ $\mathrm{CO}<\mathrm{CAOH}$.

A área superficial específica(S) dos CA foi obtida a partir das isotermas de adsorção/dessorção de $\mathrm{N}_{2}$ utilizando o método BET (Fig. 2).
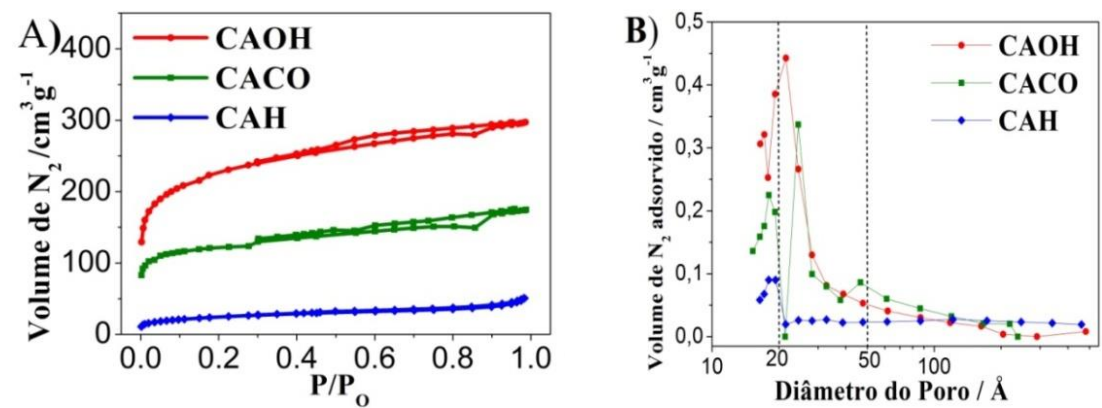

Figura 2: Isotermas de adsorção/dessorção (A) e distribuição de poros (B) das amostras CAOH, CACO e CAH.

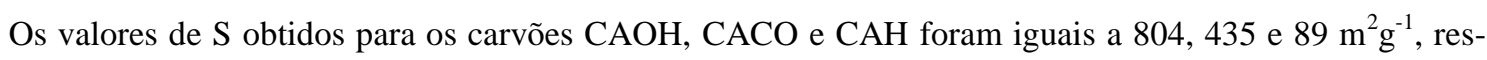
pectivamente. Estes resultados corroboram com aqueles obtidos por $\mathrm{CHN}$ (relação $\mathrm{C} / \mathrm{H}$ ). Se pode perceber que as ativações com $\mathrm{NaOH}$ e $\mathrm{CO}_{2}$ foram cerca de 9 e 4,9 vezes mais eficientes do que a realizada com o $\mathrm{H}_{3} \mathrm{PO}_{4}$. Na Figura 2B, verifica-se claramente o aumento no volume de poros dos carvões ativados com $\mathrm{CO}_{2} \mathrm{e}$ 
$\mathrm{NaOH}$, o que justifica o aumento de área superficial. Estes resultados mostram que a interação/reação dos agentes ativantes com o material precursor ocorre de forma eficiente, mesmo quando se utiliza um precursor carbonizado. Por outro lado, $\mathrm{o}_{3} \mathrm{PO}_{4}$ age de forma mais eficaz com precursores não carbonizados [11], o que resultou em uma ineficiência no processo de ativação.

A Figura 3 apresenta imagens de MEV para as amostras estudadas. A imagem da Fig.3A mostra que a FC possui estrutura fibrosa e superfície irregular contendo grandes cavidades. Por outro lado, a morfologia das amostras de CA, principalmente do $\mathrm{CAOH}$ e $\mathrm{CACO}$, apresentam cavidades menores, com formato mais arredondado e superfície mais lisa. Nota-se também que após a ativação houve redução na dimensão das partículas. Estes resultados estão coerentes com o aumento da área superficial dos carvões CAOH e CACO.

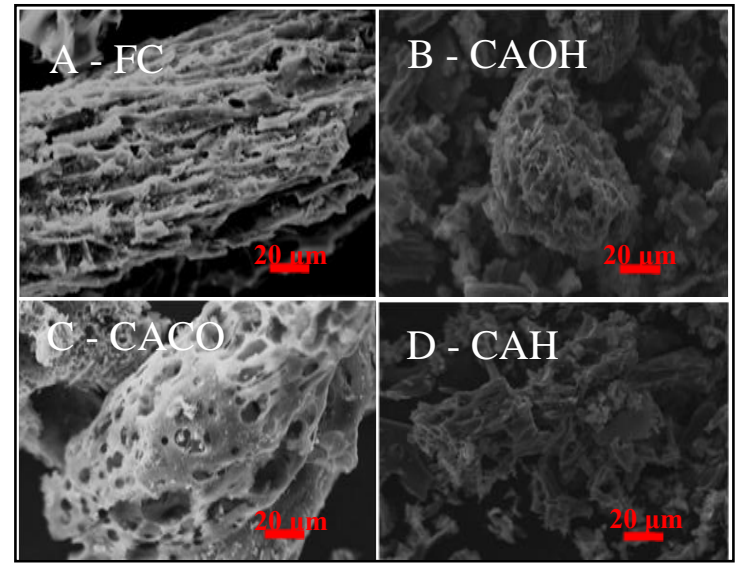

Figura 3: Imagens de MEV dos materiais estudados com ampliação de 1000x.
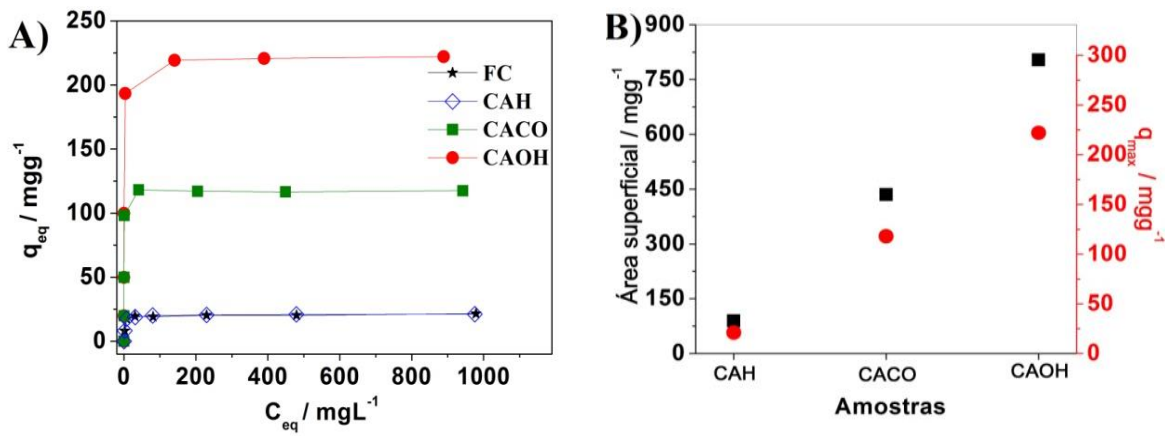

Figura 4:1 Isotermas de adsorção do $\mathrm{AM}$ pelos materiais estudados $\left(25^{\circ} \mathrm{C}, \mathrm{pH} 7\right)(\mathrm{A})$, e relação $\mathrm{S} / \mathrm{q}_{\text {máx }}(\mathrm{B})$.

A Figura 4A apresenta as isotermas de adsorção do AM pelos carvões estudados. Nota-se que as capacidades máximas de adsorção $\left(\mathrm{q}_{\max }\right)$ do AM pela FC e CAH foram praticamente iguais $\left(21 \mathrm{mgg}^{-1}\right)$ indicando que a ativação realizada com ácido não foi eficiente. Por outro lado, os valores de $\mathrm{q}_{\max }$ para o CACO e CA$\mathrm{OH}$ foram 118 e $222 \mathrm{mgg}^{-1}$, respectivamente. Na Figura 4B nota-se que a área superficial e qmax dos carvões aumentam de forma proporcional, sendo que a relação $\mathrm{S} / \mathrm{q}_{\max }$ das amostras $\mathrm{CAH}, \mathrm{CACO}$ e CAOH apresentam valores iguais a 0,236; 0,271 e $0,276 \mathrm{~m}^{2} \mathrm{mg}^{-1}$, respectivamente. Estes mostram que os carvões CACO e $\mathrm{CAOH}$ adsorvem valores muito próximos do corante $\mathrm{AM}$ por unidade de área.

\section{CONCLUSÕES}

Os resultados obtidos mostraram que os reagentes $\mathrm{NaOH}$ e $\mathrm{CO}_{2}$ foram eficientes para ativar o resíduo, fuligem de candeia, e obter carvão ativado com área superfial de 804 e $435 \mathrm{~m}^{2} \mathrm{~g}^{-1}$, respectivamente. Por outro lado, o $\mathrm{H}_{3} \mathrm{PO}_{4}$ não foi eficiente no processo de ativação. Sendo assim, conclui-se que pode-se utilizar a fuligem de candeia para obter um material com valor tecnológico e agregado, e que possui aplicação como material adsorvente como observado ao utilizar a molécula modelo de azul de metileno. 


\section{AGRADECIMENTOS}

FAPEMIG, CAPES, CNPq, UFLA,Laboratório de Microscopia Eletrônica (LME-UFLA), aos Laboratórios do Centro de Análise e Prospecção Química da UFLA (CAPQ/UFLA)e à Rede Mineira de Química (RQMG).

\section{BIBLIOGRAFIA}

[1] VANHOLME, B. et al., "Towards a carbon-negative sustainable bio-based economy", Frontier in plant science, v. 4, pp.1-17, Jun. 2013.

[2] TORQUATO, S.A., RAMOS, R.C., "BIOMASSA DA CANA-DE-AÇÚCAR E A GERAÇÃO DE BIOELETRICIDADE EM SÃO PAULO: usinas signatárias ao Protocolo Agroambiental Paulista", Informações Econômicas, v. 43, n. 5, Set. 2013.

[3] SADEZKY, A. et al., "Raman microspectroscopy of soot and related carbonaceous materials: Spectral analysis and structural information", Carbon, v. 43, n. 8, pp. 1731-42, Mar. 2005.

[4] YAASHIKAA, P.R. et al., "Advances in production and application of biochar from lignocellulosic feedstocks for remediation of environmental pollutants", Bioresource Technology, v. 292, pp. 122030, Jul. 2019.

[5] BRASIL, Lei $\mathrm{N}^{\circ} 12.305$ de 02 de agosto de 2010 - Política Nacional de Resíduos Sólidos (PNRS).Disponível em:<http://www2.mma.gov.br/port/conama/legiabre.cfm?codlegi=636>Acesso em 29 de agosto de 2019.

[6] PENIDO, E.S.; et al., "Combining biochar and sewage sludge for immobilization of heavy metals in mining soils", Ecotoxicology and Environmental Safety, v. 172, pp. 326-333, Mai. 2019.

[7] PENIDO, E.S.; et al., "Cadmium binding mechanisms and adsorption capacity by novel phosphorus/magnesium-engineered biochars", Science of the Total Environment, v. 671, pp. 1134-1143, Jun. 2019.

[8] HU, X.; GHOLIZADEH, M.; "Biomass pyrolysis: A review of the process development and challenges from initial researches up to the commercialisation stage", Journal of Energy Chemistry, v. 39, pp. 109-143, Jan. 2019.

[9] GIUSTO, L.A.R.; et al., "Preparation of Activated Carbon from Sugarcane Bagasse Soot and Methylene Blue Adsorption", Water Air Soil Pollution, pp. 228-249, Jun. 2017.

[10] GONZÁLEZ-GARCÍA, P., "Activated carbon from lignocellulosics precursors: A review of the synthesis methods, characterization techniques and applications", Renewable and Sustainable Energy Reviews, v. 82, pp. 1393-1414, Fev. 2018.

[11] SCHETTINOJÚNIOR, M. A.,et al., "Preparação e caracterização de carvão ativado quimicamente a partir da casca de arroz". Química Nova, v. 30, n. 7, pp. 1663-1668, Mar. 2007.

[12] MARSH, H., RODRÍGUEZ-REINOSO, F., Activated carbon,Amsterdam, Elsevier Science, 2006.

ORCID

Pedro Barroso Gomes

Maria Lúcia Bianchi

Fabiano Magalhães
https://orcid.org/0000-0002-5951-700X

https://orcid.org/0000-0001-8683-4222

https://orcid.org/0000-0003-1413-0810 